\title{
EFFECT OF NUTRITION SOLUTION CONCENTRATION DURING SEEDLING PREPARATION PERIOD ON SEEDLING QUALITY, YIELD AND FRUIT QUALITY IN WATERMELON (Citrullus lanatus)
}

\author{
Fawaz Al- Haji Abboud \\ Hort. Depart.-Agric. Faculty, Al- Furat Univ.
}

\begin{abstract}
We studied the effect of different concentration of nutrient solution (1NPK, 2NPK, 4NPK, 6NPK) at seedling preparation stage on seedling growth fruit quality and yield of watermelon.

As result, we found that the nutrient solution had remarkable effect on seedling survival ratio, whereas the better treatments were $2 \mathrm{NPK}$ and $4 \mathrm{NPK}$.
\end{abstract}

\section{INTRODUCTION}

Seedlings produced from sowing seeds on certain limited site called nursery, then, after reaching a specific proper length, they're transplanted in the field.

Seedling production is one of the sexual propagation methods due to the fact that seed is the source of seedling. However, some vegetable crops produce its seedlings by unisexual propagation method.

Using seedlings in the farming instead of seeds has many advantages such as:

1. To control frost, where the seedlings in green house under controlled conditions, in contrast to field conditions.

2. Possibility to use multicropping system at the same season by planting short period crops (radish, spinach, cress,..) during seedling preparation period.

3. Lowering production cost due to that seedlings occupies only limited area which saves lands and effort.

4. Increasing root system after transplanting.

5. Facilitating agricultural operations (irrigation, fertilization, thinning,..) due to limited area of the nursery.

6. Excluding type-off and infested plants.

7. To choose strong, healthy and unique plants, which fasten plant growing and increase quality and quantity yield.

8. Saving the seed amount, of remarkable importance, especially with hybrid varieties, which excels in high prices.

9. Earliness of planting date, which encourages early production.

10. Increasing production due to long period in fruitful crops and fruit number per plant.

On the other hand, transplanted plants may have a temporary retardation in the growth, if this happened, maturity will be delayed and production will decrease, and this is one of the disadvantages in the transplanting operation .

Vegetable crops differ in their transplanting ability, where some crops are considered as easy-transplanted such as (as tomato, lettuce, cabbage,..), others are less tolerant to transplanting (onion, eggplant, pepper, . .) and the last ones as difficult-transplanted such as crops of the family (Cucurbitaceae) this difference in transplanting ability attributes to its ability to compensate the missing root after taking out plants and to remain root ability to absorb water during few days following transplanting. Moreover, reduction in rooting ratio may attributes to build up Suberine and Cutin in the internal cell wall and cuticle in the cutting roots, which makes it unable to absorb water. In addition,

Fayoum J. Agric. Res. \& Dev., Vol.23, No.2, (B) July, 2009 
there are a close relationship between Suberine built up velocity and plant tolerance to transplanting, for example, accumulation happened during 3 days in bean roots, whereas tomato and cabbage roots are building through up surbine 5-6 weeks. due to:

In terms the plants of family Cucurbitaceae, transplanting is too difficult

1. Biological traits of Cucurbitaceae crops (melon, watermelon, cucumber,..), which roots can't pass the transplanting damage.

2. Poor agricultural technology and increasing effort of agricultural practices.

3. Obtained seedling quality will not represent the variety due to root damage during transplanting.

(Graves et $\mathbf{a l}, \mathbf{1 9 7 9 )}$ ) referred to the role of mineral nutrition in compensating some damaged roots.

(Rivero Rosa M. et al, 2003) confirmed that water melon irrigation by nutrient solution containing nitrogen as nitrate (6 millimose) or as ammonium (6 millimose) lowered total Fe amount in roots and leaves. Also, stated that water melon fertilization by nitrate increased plant activity without any effect on the dry material content regardless nitrogen use form.

\section{Research purpose:}

Research aimed at identifying mineral nutrition system with various concentrations, which makes seedlings adapted to a new medium conditions after transplanting. This system helps in decreasing vegetative growth, increasing nutrition stock of carbohydrates and concentrating on cellular sap which lowers freezing point and brings plants to adaptation with peripheral circumstances.

\section{MATERIALS AND METHODS}

This study was carried out in a private farm of Al-Hosainyah village (Deir Ezzor Governorate) during 2005-2006 seasons. At two successive seasons: We planted watermelon seedlings in torbic cubic $(10 \times 10 \times 4.5 \mathrm{~cm})$, which didn't contain any mineral fertilizers.

We use four nutritional solutions at different levels for NPK as clear in table (1):

Table 1. Concentration of an nutritional solution at different nutritional levels

\begin{tabular}{|l|c|c|c|}
\hline \multirow{2}{*}{$\begin{array}{c}\text { Mineral } \\
\text { nutrition }\end{array}$} & \multicolumn{3}{|c|}{ Mineral fertilization dose $\mathrm{g} / \mathrm{m}^{3}$ water } \\
\cline { 2 - 4 } & ammonium strate & super diphosphate & $\begin{array}{c}\text { potassium } \\
\text { sulphate }\end{array}$ \\
\hline 1NPK (control) & 1300 & 1000 & 1435 \\
\hline 2 NPK & 2600 & 2000 & 2870 \\
\hline 4 NPK & 5200 & 4000 & 5740 \\
\hline 6 NPK & 7800 & 6000 & 8610 \\
\hline
\end{tabular}

We irrigated torpic cubic once by nutritional solution up to saturation and $\mathrm{pH}=$ 6.5- 6.8. Irrigation was done as needed with low levels and close interval periods.

Replication number: 4

Planting date: 20 Mars 2005-2006

Plot area: $84 \mathrm{~m}^{2}(10.5 \times 8 \mathrm{~m})$

Planting dimension: $2.5 \times 2.5 \mathrm{~m}$

Fayoum J. Agric. Res. \& Dev., Vol.23, No.2, (B) July, 2009 
Row number: 2 row/treatment/replicate

Studied variety: Charleston gray

Experimental records had taken at 30 day after planting.

Statistical analysis: Randomized Complete Block Design (RCBD) was used. LSD calculated compared with control (Gomes and Gomes, 1983).

\section{RESULTS AND DISCUSSION}

Mineral nutrition level showed a remarkable effect on morphological measurements of watermelon seedlings, where on 2 NPK concentration, all seedling morphological measurements increased at remarkable level.

On the other hand, through 4 NPK and 6 NPK treatments, we observed some morphological changes: seedlings were less height and more rooting compared with seedlings irrigated by low 1 NPK, 2 NPK solutions. Also, stem, cotyledon leave stems and rips were colored by bluish-green due to anthocyanthine pigment, whereas leaves were smaller and thicker with dark green color.

On the other side, vegetative mass and stem height decreased to 1.4- 2.3 times at 4 NPK concentration. In terms of mechanical stem resistance, it decreased with increasing of solution concentration, 6 NPK treatment gave a highest resistance, while the lowest one caused by 1 and 2 NPK treatments.

Table 2. Watermelon seedling weight (gram) at different nutrition levels

\begin{tabular}{|l|l|l|}
\hline $\begin{array}{l}\text { Concentration } \\
\text { of nutrient solution NPK }\end{array}$ & $\mathbf{2 0 0 5}$ & $\mathbf{2 0 0 6}$ \\
\hline 1 (control) & $4.0 * *$ & $6.2 * *$ \\
\hline 2 & $7.2 * *$ & $9 * *$ \\
\hline 4 & $3.1 *$ & $3.8 *$ \\
\hline 6 & 1.5 & 1.8 \\
\hline LSD $_{0.05}$ & 1.68 & 1.68 \\
\hline
\end{tabular}

Table 3. Leave area , cm2/plant

\begin{tabular}{|l|l|l|}
\hline $\begin{array}{l}\text { Concentration } \\
\text { of nutrient solution NPK }\end{array}$ & $\mathbf{2 0 0 5}$ & $\mathbf{2 0 0 6}$ \\
\hline 1 (control) & $0.7^{*}$ & 0.1 \\
\hline 2 & $1.3^{* *}$ & $1.4 * *$ \\
\hline 4 & $0.6 *$ & $0.6^{*}$ \\
\hline 6 & 0.3 & 0.3 \\
\hline LSD $_{0.05}$ & 0.32 & 0.47 \\
\hline
\end{tabular}

Table 4. Leave number, leave/plant

\begin{tabular}{|l|l|l|}
\hline $\begin{array}{l}\text { Concentration } \\
\text { of nutrient solution NPK }\end{array}$ & $\mathbf{2 0 0 5}$ & $\mathbf{2 0 0 6}$ \\
\hline 1 (control) & $3 * *$ & $3 * *$ \\
\hline 2 & $4 * *$ & $4 * *$ \\
\hline 4 & $3 * *$ & $3 * *$ \\
\hline 6 & $2 * *$ & $2 *$ \\
\hline LSD $_{0.05}$ & 1.3 & 1.3 \\
\hline
\end{tabular}

According to table 4, the highest concentration produced good seedlings (2- 3 leaves) compared with control treatment.

Fayoum J. Agric. Res. \& Dev., Vol.23, No.2, (B) July, 2009 
Table 5. Watermelon seedling height, $\mathrm{cm}$

\begin{tabular}{|l|l|l|}
\hline $\begin{array}{l}\text { Concentration } \\
\text { of nutrient solution NPK }\end{array}$ & $\mathbf{2 0 0 5}$ & $\mathbf{2 0 0 6}$ \\
\hline 1 (control) & $4.8^{*}$ & $5.8^{* *}$ \\
\hline 2 & $7.0 * *$ & $7.5 * *$ \\
\hline 4 & $4.3 *$ & $4.6 *$ \\
\hline 6 & 3.7 & 3.7 \\
\hline LSD $_{0.05}$ & 1.34 & 1.0 \\
\hline
\end{tabular}

Table 6. Stem diameter, $\mathrm{cm}$

\begin{tabular}{|l|l|l|}
\hline $\begin{array}{l}\text { Concentration } \\
\text { of nutrient solution NPK }\end{array}$ & $\mathbf{2 0 0 5}$ & $\mathbf{2 0 0 6}$ \\
\hline 1 (control) & $0.45 *$ & $0.47 *$ \\
\hline 2 & $0.46 *$ & $0.54 * *$ \\
\hline 4 & $0.42 *$ & $0.42 *$ \\
\hline 6 & 0.34 & 0.36 \\
\hline LSD $_{0.05}$ & 0.059 & 0.101 \\
\hline
\end{tabular}

Table 7. Germination percentage, $\%$

\begin{tabular}{|l|l|l|}
\hline $\begin{array}{l}\text { Concentration } \\
\text { of nutrient solution NPK }\end{array}$ & $\mathbf{2 0 0 5}$ & $\mathbf{2 0 0 6}$ \\
\hline 1 (control) & $92 * *$ & $84 * *$ \\
\hline 2 & $91 * *$ & $80 * *$ \\
\hline 4 & $93 * *$ & $79 * *$ \\
\hline 6 & $83^{* *}$ & $70 * *$ \\
\hline LSD $_{0.05}$ & 5.4 & 5.64 \\
\hline
\end{tabular}

From table 7, germination percentage was high at all nutrient concentrations, indicating to the positive effect on germination percentage.

Table 8. Seedling survive percentage

\begin{tabular}{|l|l|l|}
\hline $\begin{array}{l}\text { Concentration } \\
\text { of nutrient solution NPK }\end{array}$ & $\mathbf{2 0 0 5}$ & $\mathbf{2 0 0 6}$ \\
\hline 1 (control) & $73 * *$ & $92 * *$ \\
\hline 2 & $77^{* *}$ & $94 * *$ \\
\hline 4 & $81 * *$ & $97 * *$ \\
\hline 6 & $7.3 * *$ & 2.97 \\
\hline LSD $_{0.05}$ & 2.97 & 7.3 \\
\hline
\end{tabular}

Here, nutrient solution concentration played a remarkable role on seedling survivel ratio, where 4 and 6 NPK treatments showed highest ratios after transplanting, which increase dry mater, chlorophyll and nutrients in seedlings as well as rootlets at the higher root zone and good root system, which explained by scarcity of mechanical damage during transplanting (Boaw 1984).

Nevertheless, we observed death of leaves on 10-15 days following transplanting at 1 and 2 NPK treatments, while at 4 and 6 NPK treatments, we observed death of the lowest two leaves, and after 25-30 days with high concentrations of 4 and $6 \mathrm{NPK}$, seedlings grew better compared with those irrigated by low concentrations ( 1 and 2 NPK). Also, there're differences in production when solution concentration changed, the highest yield was obtained by 4 and 6 NPK.

Fayoum J. Agric. Res. \& Dev., Vol.23, No.2, (B) July, 2009 
Table 9. Fruit number, fruit/plant

\begin{tabular}{|l|l|l|}
\hline $\begin{array}{l}\text { Concentration } \\
\text { Of nutrient solution NPK }\end{array}$ & $\mathbf{2 0 0 5}$ & $\mathbf{2 0 0 6}$ \\
\hline 1 (control) & $1^{*}$ & $1^{*}$ \\
\hline 2 & $2^{*}$ & $2^{*}$ \\
\hline 4 & $2^{*}$ & $2^{*}$ \\
\hline 6 & $2^{*}$ & $2^{*}$ \\
\hline LSD $_{0.05}$ & 0.98 & 0.98 \\
\hline
\end{tabular}

Table 10. Total yield under different nutrition levels, ton/ $1000 \mathrm{~m}^{2}$

\begin{tabular}{|l|l|l|}
\hline $\begin{array}{l}\text { Concentration } \\
\text { of nutrient solution NPK }\end{array}$ & $\mathbf{2 0 0 5}$ & $\mathbf{2 0 0 6}$ \\
\hline 1 (control) & $1.23^{* *}$ & $1.96^{* *}$ \\
\hline 2 & $1.63 * *$ & $2.4 * *$ \\
\hline 4 & $1.97 * *$ & $2.74 * *$ \\
\hline 6 & $2.11 * *$ & $2.61 * *$ \\
\hline LSD $_{0.05}$ & 0.26 & 0.33 \\
\hline
\end{tabular}

Table 11. Dry matter percentage, $\%$

\begin{tabular}{|l|l|l|}
\hline $\begin{array}{l}\text { Concentration } \\
\text { Of nutrient solution NPK }\end{array}$ & $\mathbf{2 0 0 5}$ & $\mathbf{2 0 0 6}$ \\
\hline 1 (control) & 8.5 & 8.0 \\
\hline 2 & 8.5 & 8.8 \\
\hline 4 & 9.0 & 8.0 \\
\hline 6 & 8.0 & 8.8 \\
\hline
\end{tabular}

F calculated $0.288<\mathrm{F}$ tabulated 6.39

According to Table 11, fruit dry matter didn't affected by concentration of nutrient solution (Simonne, et al, 2005).

Table 12. Sugar ratio in watermelon fruits, $\%$

\begin{tabular}{|l|l|l|}
\hline $\begin{array}{l}\text { Concentration } \\
\text { Of nutrient solution NPK }\end{array}$ & $\mathbf{2 0 0 5}$ & $\mathbf{2 0 0 6}$ \\
\hline 1 (control) & 7.8 & 7.2 \\
\hline 2 & 7.4 & 6.8 \\
\hline 4 & 8.1 & 6.3 \\
\hline 6 & 6.8 & 7.2 \\
\hline
\end{tabular}

F calculated $(0.181)<\mathrm{F}$ tabulated $(6.39)$

According to Table 12, fruit sugar ratio didn't affected by concentration of nutrient solution.

Stated that carbohydrate concentration in water melon fruits increased by 6 folds after flower pollination, this increase was associated with increase in invertase activity, which have a high concentration in pollinated ovaries.

Table 13. Vitamin $C$ content in watermelon fruits, $\%$

\begin{tabular}{|l|l|l|}
\hline $\begin{array}{l}\text { Concentration } \\
\text { Of nutrient solution NPK }\end{array}$ & $\mathbf{2 0 0 5}$ & $\mathbf{2 0 0 6}$ \\
\hline 1 (control) & 4.2 & 5.1 \\
\hline 2 & 4.4 & 4.6 \\
\hline 4 & 4.4 & 7.1 \\
\hline 6 & 4.3 & 6.1 \\
\hline
\end{tabular}

F calculated $(0.455)<\mathrm{F}$ tabulated $(6.39)$

Fayoum J. Agric. Res. \& Dev., Vol.23, No.2, (B) July, 2009 
Table 14. Nitrate content in watermelon fruits, $\mathrm{mg} / \mathrm{kg}$ dry matter

\begin{tabular}{|l|l|l|}
\hline $\begin{array}{l}\text { Concentration } \\
\text { Of nutrient solution NPK }\end{array}$ & $\mathbf{2 0 0 5}$ & $\mathbf{2 0 0 6}$ \\
\hline 1 (control) & 39 & 70 \\
\hline 2 & 40 & 66 \\
\hline 4 & 37 & 63 \\
\hline 6 & 32 & 71 \\
\hline
\end{tabular}

F calculated (0.015) < F tabulated (6.39)

According to Tables 11 to 14 , the calculated $\mathrm{F}$ was less than tabulated F, indicating that no significant differences were noticed between some traits (e.g. $\%$ dry matter, \% sugar, \% vitamin $\mathrm{C}$ and nitrate fruit content) were found at different concentrations, so no need to LSD test.

\section{Recommendations}

The strongest, good -rooted seedlings obtained by 4 NPK and 6 NPK.

1. At $4 \mathrm{NPK}$, high germination ratio and seedling survival ratio were obtained.

2. 4 NPK concentration helped increasing yield in watermelon crop.

\section{REFERENCES}

بوراس متيادى وحداد سليم ( • 99 (1)، الزر اعة المحمية، الجزء العملي، منشور ات جامعه دمشق.

Boaw, W. (1980). The establishment and yield of vegetable crop grown from blocks designed for automatic transplanting export. Hortic. London. 31.p.26-34.

Graves, B. (1979). Vegetable transplant vegetal. Growers news.34.s.1-4.

Hassan, A. M. (1991). Principles of vegetable production. Al-Dar Al-Arabia Publisher Cairo.

Khalf Alla, E. (1990). The principles of vegetable production. Alexandria University Publisher.

Ramzi, I. K. (1951). Vegetable production. Anglo Masrya Maktabh Cairo.

$$
\begin{aligned}
& \text { تأثير تركيز المحاليل المغذية أثناء إنتاج البادرات على حيوية البادرات } \\
& \text { والمحصول وجودة الثمار في البطيخ البخاء التيخ } \\
& \text { فواز الحاجي عبود }
\end{aligned}
$$

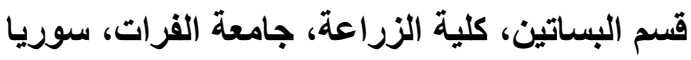

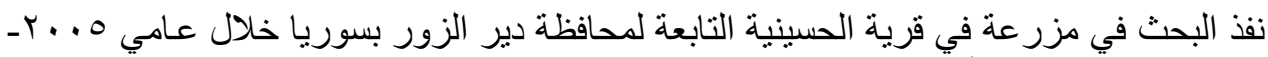

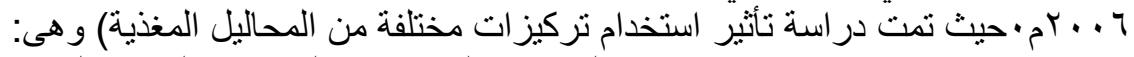

$$
\begin{aligned}
& \text { (1NPK-2NPK-4NPK-6NPK) }
\end{aligned}
$$

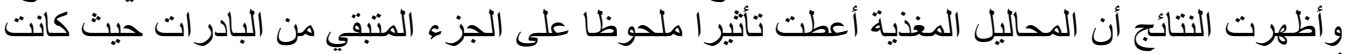

$$
\begin{aligned}
& \text { أحسن معاملتين هما 2NPK-4NPK بالترتيب. }
\end{aligned}
$$

Fayoum J. Agric. Res. \& Dev., Vol.23, No.2, (B) July, 2009 